\title{
The Smell of Success?-The Impact of Perfume-Gender Congruency on Ratings of Attraction and the Halo Effect
}

\author{
Radost Marinova, Mark Moss \\ Department of Psychology, Faculty of Health and Life Sciences, Northumbria University, Newcastle upon Tyne, \\ UK \\ Email: mark.moss@unn.ac.uk
}

Received 25 August 2014; revised 10 September 2014; accepted 25 September 2014

Copyright (C) 2014 by authors and Scientific Research Publishing Inc.

This work is licensed under the Creative Commons Attribution International License (CC BY). http://creativecommons.org/licenses/by/4.0/

(c) (i) Open Access

\begin{abstract}
Research into the concept of attraction has indicated the existence of olfactory influences that go beyond pheromonal level. Synthetic fragrances have been found to affect not only perception of attraction, but also attribution of beauty-unrelated intrinsic characteristics. The present study aimed to examine the impact of perfume-gender congruency on ratings of attractiveness and any perfume-generated halo effect. Male faces categorized as low, medium and high attractiveness were rated by 36 heterosexual female students across six domains: attractive, reliable, outgoing, intelligent, wealthy and socially competent. Ratings were made in the presence of a female perfume (incongruent condition), male perfume (congruent condition) or a no perfume control condition, with participants randomly allocated to produce three groups of equal size. The results indicated that 1) attractiveness generated a significant halo effect; 2) the male perfume did not significantly enhance perception of attractiveness compared to the female perfume; and 3) the gender-congruent fragrance heightened attribution of "halo" characteristics. These results indicate that gender congruent perfume can impact positively on first impressions beyond attractiveness, and are discussed in terms of possible mechanisms and implications.
\end{abstract}

\section{Keywords}

Perfume, Congruence, Attractiveness, Halo Effect

\section{Introduction}

Attraction is "the feeling of being drawn to one or more other individuals... and the extent to which any one in-

How to cite this paper: Marinova, R. and Moss, M. (2014) The Smell of Success?-The Impact of Perfume-Gender Congruency on Ratings of Attraction and the Halo Effect. Advances in Chemical Engineering and Science, 4, 491-502.

http://dx.doi.org/10.4236/aces.2014.44051 
dividual is attractive to or liked by others” [1]. Whilst no objective measure for the construct has been established, Bull and Rumsey [2] envisioned it as a product of the consensus amongst raters. It is uniformly seen as an honest indicator of reproductive fitness and genetic value, and its importance is reflected in the favourable responses evoked in others [3] People often engage in "peacocking" behaviours, such as grooming, investing in cosmetics and dressing in carefully selected clothes, in attempt to manipulate perceived attraction levels [4].

A link between perceived physical beauty and the attribution of seemingly unrelated favourable characteristics resulting from this initial positive perception, the "halo effect" [5] is well established. Attractive individuals are immediately endowed with a variety of favourable personal attributes including being intelligent [6], warm, socially skilled, exciting, polite, extraverted [7], reciprocative and trustworthy [8], as a consequence of this "beautiful-is-good" stereotype. However, actual relationships between mental ability and personality traits, and attractiveness are in fact negligible [9], suggesting that at least some of these attributions are erroneous. Nevertheless, the stereotype persists.

The importance of the halo effect is represented in the extent to which it affects numerous domains. Physically attractive pupils tend to receive more help from their teachers and more positive judgements regarding their intellectual ability and future potential [10]. Later in life, they experience more favourable job-related outcomes. Occupational literature strongly supports the existence of the halo effect; the emergence of socially desirable features like competence and cooperation [11] [12] amass to applicant favourability. Thus, attaching a highly attractive photograph to a résumé leads to an elevation of judgement of its quality and value [13], hiring recommendations and predicted job success, irrespective of the interviewer's level of professionalism or experience [14]. Further workplace implications are reflected in the wage gap benefitting good-looking individuals, with attractiveness being reported as a more influential determinant of earnings than ability [15].

A number of biological and environmental factors have been found to facilitate attraction. From a biological perspective, pheromones are a well-established intraspecific signalling system of genetic quality, fertility and sexual readiness across most species, of which humans are not an exception. Despite the decrease in olfactory acuity in humans, the ability to detect them in amounts as small as 100 picograms remains unaffected [16]. Pheromones act as natural attractants of the opposite sex. This effect is evident in the results of an experimental study by Kirk-Smith and Booth [17]. An androsenone (male pheromone) treated chair was placed in a dentists waiting room and the frequency with which men and women used it was recorded. Women tended to gravitate towards the chair, whilst the odourless cue had the opposite effect on men. Although it would be expected that close physical proximity would be required for detection of the pheromone, a similar study conducted in a wash room found the same effect [18]. The authors suggest that the pheromone elicits feelings of safety and comfort in females, which highlights the importance of the olfactory sense in cognition and perception. Additionally, in the presence of androstenol (pheromone present in fresh male sweat) females rated male targets as more attractive, friendly, sexy and warm [19], implicating the generation of a halo effect.

In a different arena, retailers constantly strive to make products more attractive to customers in order to boost sales, and this is often achieved through the manipulation of environmental odour. Specifically the congruency between products and ambient smell in retail environments has been shown to impact on shopping tendencies. Diffusing juniper berry (a primary ingredient of gin) aromas effectively boosted sales rates for alcohol [20]. Congruency between the target consumers and the smell within the environment may be of even greater importance. Spangenberg, Sprott, Grohmann \& Tracy [21] demonstrated a doubling in women's clothes sales when feminine scents were diffused in the air. The same effect was observed for men in the presence of masculine odours, which might highlight the importance of employing a gender-matching fragrance in order to bring about favourable responses and behaviours.

People often engage in personal odorizing and deodorizing rituals for a number of reasons. These include avoidance of negative judgement and moral stigmatization resulting from poor body odour, attempting to obtain the desired etiquette, for overall sense of personal appeal and confidence [22]. The perfume industry has recognized the importance of olfactory cues and heavily relies on this unquestionable desire to be liked and accepted. Perfume advertisements go as far as to suggest that their product enhances sexual appeal, often implying power, success and charm [23]. They allege that explicitly male or female perfumes virtually guarantee the wearer the acceptance and liking of others [24]. Whilst these claims may seem far-fetched, research has, in fact, provided some support and demonstrated scent-elicited positive responses.

In Baron's [25] experiment participants interacted briefly with a confederate who either wore a perfume (female fragrance "Jungle Gardenia") or did not, and were subsequently asked to rate them. Results indicated posi- 
tive shifts in evaluation of the perfume-wearing confederate for both attractiveness and socially desirable attributes, in comparison to the one who did not wear a scent. Subsequently, in 1990, Kirk-Smith and Booth [26] investigated whether this effect extended to evaluations of individuals who were not physically present during or prior to the assessment. Examining the potential cross-modal effect of pleasant ambient scent on judgement of attractiveness of half-torso male and female photographs, the authors found a significant positive effect of female perfume "Shalimar" on perception of how "sexy" and "soft" the individual is, relative to a no perfume condition. An elevation in mood was also reported; Kirk-Smith and Booth proposed that this mood shift was the primary cause of the enhanced perceptions. However, a banana essence produced no such effect, despite being rated as equally pleasant in a preliminary study. This reiterates the necessity for congruency between the target and the smell as an individual is much more likely to be associated with a perfume than a food-related scent.

The visual-olfactory perceptual interaction was later addressed by Capparuccini, Berrie and Mazzatenta [27]. In a repeated measures design, a mixed-gender sample of raters visually assessed passport-sized photographs of male and female faces across a range of qualities during non-conscious administration of male (Givenchy) or female (Angel \& Demon) perfumes. The focal rating traits were categorized into sexually-neutral (confidence and familiarity), moderately sexually-related (liking and irritability) and highly sexually-related (charm, pleasantness, intensity, beauty, sexual interest and sexual attraction) groups. Results indicated a significant impact on the latter two categories with higher ascriptions of those traits to both sexes as a result of the olfactory stimulation. However, the findings only applied to trials where the sex of the facial stimuli and the gender-specific perfume corresponded, connoting the importance of congruency in this cross-modal interaction.

The current study will focus on the effect of the congruency between sex of target stimuli and gender-specific scent on ratings of facial attractiveness. There will be a focus on the halo effect, so as to examine whether perfume can enhance the effect attractiveness alone does, and alter perception of the individual. Based on existing literature three outcomes are hypothesized. Firstly, it is expected that more attractive stimuli will generate a halo effect compared to less attractive stimuli irrespective of any impact of perfume. Secondly, congruency between the gender-specific perfume and the sex of the facial stimuli will increase the ratings of attractiveness for all stimuli, although whether this increase will be the same for all categories of stimuli attractiveness cannot be predicted. Thirdly, it is proposed that perfume-congruency will enhance the halo effect for the stimuli when compared the incongruent perfume and no perfume conditions.

\section{Method}

The following procedure received ethical approval from the Department of Psychology Ethics Committee at Northumbria University.

\subsection{Design}

A 3 (perfume exposed to: male vs. female vs. no perfume; between subjects) $\times 3$ (level of attractiveness of faces: low vs. medium vs. high; within subjects) mixed factorial design was employed for the purpose of the study. The dependent variables were participants' ratings on how attractive, reliable, outgoing, intelligent, wealthy and socially competent the photographed males are, with the latter five adjectives being measures of the halo effect.

\subsection{Participants}

An opportunity sample consisting of 36 female Social Sciences undergraduate students from the North East of England formed three groups of 12 people for each condition. All participants were of white Caucasian ethnic origin and similar age $(\mathrm{M}=20.83, \mathrm{SD}=0.90$ in male perfume condition; $\mathrm{M}=21.75, \mathrm{SD}=2.24$ in female perfume condition; $\mathrm{M}=20.08, \mathrm{SD}=0.86$ in control condition). Fourteen participants were single whilst the other 22 reported currently being in a relationship. The exclusion criteria only allowed for heterosexual females with normal or corrected-to-normal sight, no olfactory dysfunction, and in the absence of an active cold or flu, to take part. All participants were unpaid volunteers.

\subsection{Materials}

A PowerPoint presentation with 5 "unattractive", 5 "average" and 5 "attractive” female faces, (based on the results from a pilot study) was utilized as stimuli. The faces exhibited a neutral expression or a closed-mouth smile. 
Humidity and temperature in the room were measured with a digital thermometer. Two commercially successful perfumes were used for the experimental conditions: Hugo Boss-In Motion for men and Ghost-Ghost for women. Participants recorded their ratings for each face on a 10 point scale for both attractiveness $(1=$ very unattractive, $10=$ very attractive $)$ and each of the five "halo" characteristics $(1=$ not at all, $10=$ very much so $)$.

\subsection{Procedure}

Two minutes prior to the participant's arrival, the room ( $5 \mathrm{~m}$ length $\times 2.2 \mathrm{~m}$ width $\times 2.4 \mathrm{~m}$ height) was set up so as to construct one of two experimental conditions, by spraying male or female perfume (four spritzes) in the air. In a control condition no perfume was used. Assignation of participants to conditions was randomized. The thermometer was placed on the desk near the laptop so as to measure the temperature experienced by the participant as accurately as possible. The presentation started with a slide containing a set of instructions clarifying the scoring process. At the press of the "spacebar" key participants were able to move on to the next slide which displayed a different face stimulus, once a rating had been provided. The experimenter was not present in the room for the duration of the experiment (approximately 15 - 20 minutes). Upon completion participants were thanked and debriefed as to the true purpose of the study.

The average humidity in the room was measured to be $42.2 \%$ (lower bound $=39 \%$; upper bound $=45 \%$ ). Room temperature was maintained between $15.7^{\circ} \mathrm{C}$ and $17.7^{\circ} \mathrm{C}$, and fluctuated by no more than 0.3 degrees across any one trial. The distance between the chair and laptop was kept consistent across trials at approximately $80 \mathrm{~cm}$, which is the distance at which person-to-person interaction is expected to take place under normal circumstances. In order to reduce contamination between fragrances, the window was left open for at least 12 hours when alternating between experimental conditions.

\section{Results}

Data were analysed using $3 \times 3$ mixed factorial Anovas with fragrance condition as the between groups factor with three levels (congruent, incongruent and no perfume) and attractiveness of facial stimuli as the within groups factor with three levels (high, medium and low attractiveness). Post hoc comparisons were calculated using the Tukey method for the between groups factor and the Bonferroni method for the within groups factor.

\subsection{Attractiveness}

The descriptive statistics for ratings of attractiveness assigned to the images in the three stimuli categories and three perfume conditions are presented in Table 1 below.

The analysis revealed a significant main effect of stimuli attractiveness category on the ratings of attractiveness given to the images $\mathrm{F}(2,66)=426.317, \mathrm{p}<0.001$, partial eta squared $=0.928$. Pairwise comparisons revealed that the high attractive category (mean $=6.70$ ) gained significantly higher ratings than the medium category (mean $=4.10$ ) which gained significantly higher ratings than the low category (mean $=1.89$ ), $\mathrm{p}<0.001$ in all cases. This clearly indicates that the categories of images used accurately reflected differences in attractiveness as designed.

No significant main effect of perfume on ratings of attractiveness was found $F(2,33)=2.006, p=0.151$, partial eta squared $=0.108$.

Interestingly, a significant attractiveness by perfume interaction effect was identified, $F(4,66)=3.720, \mathrm{p}=$ 0.009, partial eta squared $=0.184$. This is represented in Figure 1 . As can be seen the incongruent per fume (Ghost)

Table 1. Means (Standard Deviations) of ratings of “Attractiveness" of high, medium and low attractiveness image categories under the three perfume conditions, $\mathrm{N}=36$.

\begin{tabular}{ccccc} 
& High & Medium & Low & Total \\
\hline Congruent & $6.88(0.82)$ & $4.73(1.17)$ & $2.10(1.24)$ & $4.57(1.08)$ \\
Incongruent & $7.03(0.99)$ & $3.68(0.91)$ & $1.63(0.57)$ & $1.95(0.87)$ \\
No Perfume & $6.18(0.83)$ & $3.88(0.78)$ & $1.89(0.93)$ \\
Total & $6.70(0.94)$ & $4.10(1.05)$ & $4.01(0.83)$ \\
\hline
\end{tabular}




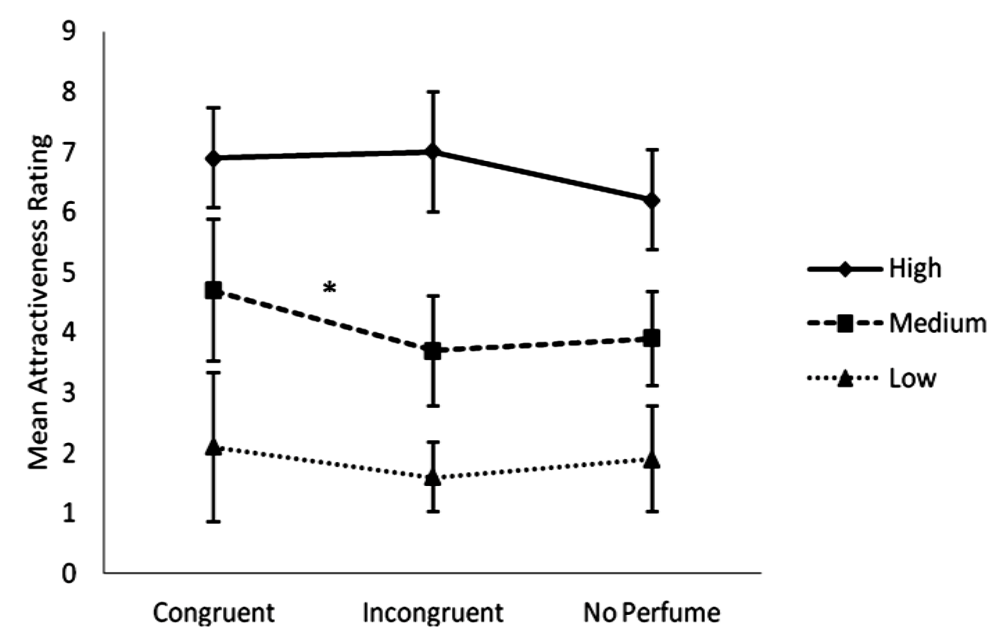

Figure 1. Mean attractiveness ratings gained by each stimulus attractiveness category in each perfume condition. Error bars depict standard deviations. *denotes significant pairwise comparison from analysis of simple effects of perfume type for each category of stimuli attractiveness.

is associated with the lowest ratings of attractiveness for the low and mediums attractiveness categories but that this is not the case for the high attractiveness category where it is couple with the highest ratings. However, an analysis of simple effects revealed that the only significant difference between conditions across the different attractiveness categories was between the two perfume conditions in the medium category, $\mathrm{p}=0.031$, where the congruent perfume produced the higher ratings.

\subsection{Halo Effect}

\subsubsection{Reliable}

Descriptive statistics for the ratings of the trait "reliable" by participants for the three categories of image in the three perfume conditions are presented in Table 2.

The analysis revealed a significant main effect of stimuli attractiveness on ratings on this scale $\mathrm{F}(2,66)=$ 60.942, $\mathrm{p}<0.001$, partial eta squared $=0.649$. Pairwise comparisons indicated that ratings for the high attractiveness images (mean $=6.12$ ) were significantly higher than for the medium attractiveness images (mean $=4.87$ ) which in turn were significantly higher than for the low attractiveness condition (mean $=3.49$ ), $p<0.001$ in all cases.

No significant main effect of perfume condition was found for the "reliable" variable, $F(2,33)=2.406, \mathrm{p}=$ 0.106 , partial eta squared $=0.127$.

The attractiveness by perfume condition interaction effect approached but did not reach statistical significance $\mathrm{F}(4,66)=2.295, \mathrm{p}=0.068$, partial eta squared $=0.122$.

\subsubsection{Outgoing}

Descriptive statistics for the ratings of the trait "outgoing” by participants for the three categories of image in the three perfume conditions are presented in Table 3.

The analysis revealed a significant main effect of stimuli attractiveness on ratings on the "outgoing" scale $F(2$, 66 ) $=190.080, \mathrm{p}<0.001$, partial eta squared $=0.852$. Pairwise comparisons indicated that ratings for the high attractiveness images (mean $=7.41$ ) were significantly higher than for the medium attractiveness images (mean $=6.01$ ) which in turn were significantly higher than for the low attractiveness condition (mean $=3.78$ ), $\mathrm{p}<$ 0.001 in all cases.

A significant main effect of perfume condition was found for the "outgoing" variable, $F(2,33)=6.719, \mathrm{p}=$ 0.004 , partial eta squared $=0.289$. Pairwise comparisons indicated that the congruent perfume (mean $=6.37$ ) produced significantly higher ratings than both the incongruent perfume (mean $=5.53$ ), $\mathrm{p}=0.032$, and the no perfume conditions (mean $=5.29$ ), $\mathrm{p}=0.004$. 
Table 2. Means (Standard Deviations) of ratings on the trait "Reliable" for high, medium and low attractiveness image categories under the three perfume conditions, $\mathrm{N}=36$.

\begin{tabular}{ccccc}
\hline & High & Medium & Low & Total \\
\hline Congruent & $6.80(1.41)$ & $5.50(1.31)$ & $3.30(0.99)$ & $5.20(1.24)$ \\
Incongruent & $5.95(1.16)$ & $4.48(0.85)$ & $3.68(1.33)$ & $4.71(1.11)$ \\
No Perfume & $5.60(0.93)$ & $4.62(0.76)$ & $3.48(1.07)$ & $4.57(0.87)$ \\
Total & $6.12(1.26)$ & $4.87(1.07)$ & $3.49(1.12)$ & \\
\hline
\end{tabular}

Table 3. Means (Standard Deviations) of ratings on the trait "Outgoing” for high, medium and low attractiveness image categories under the three perfume conditions, $\mathrm{N}=36$.

\begin{tabular}{ccccc}
\hline & High & Medium & Low & Total \\
\hline Congruent & $7.93(1.01)$ & $6.48(0.79)$ & $4.68(1.14)$ & $5.53(0.98)$ \\
Incongruent & $7.53(0.92)$ & $5.68(0.82)$ & $3.38(1.08)$ & $6.37(0.94)$ \\
No Perfume & $6.77(0.81)$ & $5.85(0.97)$ & $3.27(1.29)$ & $5.29(1.02)$ \\
Total & $7.41(1.01)$ & $6.01(0.91)$ & $3.78(1.31)$ & \\
\hline
\end{tabular}

The attractiveness by perfume condition interaction effect did not reach statistical significance $F(4,66)=$ 1.864, $\mathrm{p}=0.127$, partial eta squared $=0.101$.

\subsubsection{Intelligent}

Descriptive statistics for the ratings of the trait "intelligent" by participants for the three categories of image in the three perfume conditions are presented in Table 4.

The analysis revealed a significant main effect of image attractiveness on ratings on the "intelligent" scale $F(2$, $66)=9.097, \mathrm{p}<0.001$, partial eta squared $=0.216$. Pairwise comparisons indicated that ratings for the high attractiveness images (mean $=6.53$ ) were significantly higher than for the medium attractiveness images (mean = 6.044), $\mathrm{p}=0.042$, and the low attractiveness condition (mean $=5.583$ ), $\mathrm{p}=0.003$. The medium and low attractiveness images did not differ significantly on this scale, $\mathrm{p}=0.101$.

A significant main effect of perfume condition was also found for the "intelligent" variable, $F(2,33)=5.230$, $\mathrm{p}=0.011$, partial eta squared $=0.241$. Pairwise comparisons revealed that the congruent perfume $($ mean $=6.57)$ produced significantly higher ratings than the no perfume condition (mean $=5.68$ ), $p=0.011$. The difference between the congruenat and incongruent perfume (mean $=5.92$ ) approached but did not reach statistical significance, $\mathrm{p}=0.087$. The incongruent and no perfume conditions did not differ, $\mathrm{p}=1$.

The attractiveness by perfume condition interaction effect did not reach statistical significance $F(4,66)=$ $1.151, \mathrm{p}=0.340$, partial eta squared $=0.065$.

\subsubsection{Wealthy}

Descriptive statistics for the ratings of the trait "wealthy" by participants for the three categories of image in the three perfume conditions are presented in Table 5.

The analysis revealed a significant main effect of stimuli attractiveness on ratings on the "wealthy" scale $\mathrm{F}(2$, $66)=28.704, p<0.001$, partial eta squared $=0.465$. Pairwise comparisons indicated that ratings for both the high attractiveness images (mean $=5.983)$ and the medium attractiveness images (mean $=5.494)$ were significantly higher than for the low attractiveness condition (mean $=4.456$ ), $\mathrm{p}<0.001$ in each case. The high and medium attractiveness images did not differ significantly on this variable, $\mathrm{p}=0.090$.

A significant main effect of perfume condition was also found for the "wealthy" variable, $F(2,33)=5.247, p$ $=0.011$, partial eta squared $=0.241$. Pairwise comparisons revealed that the congruent perfume condition (mean $=5.96$ ) produced significantly higher ratings than both the incongruent perfume (mean $=4.98$ ), $\mathrm{p}=0.024$, and the no perfume conditions (mean $=4.99) \mathrm{p}=0.026$. The incongruent perfume and no perfume conditions did not differ, $\mathrm{p}=1$. 
Table 4. Means (Standard Deviations) of ratings on the trait "Intelligent" for high, medium and low attractiveness image categories under the three perfume conditions, $\mathrm{N}=36$.

\begin{tabular}{ccccc}
\hline & High & Medium & Low & Total \\
\hline Congruent & $6.98(1.12)$ & $6.85(1.27)$ & $5.87(1.07)$ & $6.57(1.15)$ \\
Incongruent & $6.63(0.97)$ & $5.63(1.04)$ & $5.48(0.85)$ & $5.92(0.95)$ \\
No Perfume & $5.98(1.23)$ & $5.65(0.57)$ & $5.40(1.05)$ & $5.67(0.95)$ \\
Total & $6.53(1.16)$ & $6.04(1.13)$ & $5.58(0.99)$ & \\
\hline
\end{tabular}

Table 5. Means (Standard Deviations) of ratings on the trait "Wealthy" for high, medium and low attractiveness image categories under the three perfume conditions, $\mathrm{N}=36$.

\begin{tabular}{ccccc}
\hline & High & Medium & Low & Total \\
\hline Congruent & $6.58(1.42)$ & $6.18(1.14)$ & $5.10(1.14)$ & $5.95(1.23)$ \\
Incongruent & $5.71(0.92)$ & $5.17(0.72)$ & $4.07(1.12)$ & $4.98(0.92)$ \\
No Perfume & $5.65(1.24)$ & $5.13(0.86)$ & $4.20(1.21)$ & $4.99(1.10)$ \\
Total & $5.98(1.25)$ & $5.49(1.02)$ & $4.46(1.22)$ & \\
\hline
\end{tabular}

The attractiveness by perfume condition interaction effect did not reach statistical significance $F(4,66)=$ $0.068, \mathrm{p}=0.991$, partial eta squared $=0.004$.

\subsubsection{Socially Competent}

Descriptive statistics for the ratings of the trait "socially competent" by participants for the three categories of image in the three perfume conditions are presented in Table 6.

The analysis revealed a significant main effect of stimuli attractiveness on ratings on this scale $\mathrm{F}(2,66)=$ 92.090, $\mathrm{p}<0.001$, partial eta squared $=0.736$. Pairwise comparisons indicated that ratings for the high attractiveness images (mean $=7.49$ ) were significantly higher than for the medium attractiveness images $($ mean $=6.29$ ) which in turn were significantly higher than for the low attractiveness condition (mean $=4.30$ ), $p<0.001$ in all cases.

A significant main effect of perfume condition was also found for the "socially competent" variable, $F(2,33)$ $=4.870, \mathrm{p}=0.014$, partial eta squared $=0.228$. Pairwise comparisons indicated that the congruent perfume (mean $=6.59$ ) produced significantly higher ratings than the no perfume condition (mean $=5.58$ ), $p=0.013$. No difference was found between the congruent perfume and incongruent perfume (mean $=5.92$ ) conditions, $\mathrm{p}=$ 0.146 , or between the incongruent perfume and no perfume conditions, $\mathrm{p}=0.952$.

The attractiveness by perfume condition interaction effect did not reach statistical significance $F(4,66)=$ $0.975, \mathrm{p}=0.427$, partial eta squared $=0.056$.

\subsection{Potentially Confounding Variables}

Previous research has identified self-rated attractiveness, week of menstrual cycle and relationship status as confounding variables in perception of attractiveness. A Pearson correlation indicated no significant correlation

Table 6. Means (Standard Deviations) of ratings on the trait "Socially Competent” for high, medium and low attractiveness image categories under the three perfume conditions, $\mathrm{N}=36$.

\begin{tabular}{ccccc} 
& High & Medium & Low & Total \\
\hline Congruent & $8.10(1.37)$ & $6.87(1.51)$ & $4.80(1.51)$ & $3.97(1.14)$ \\
Incongruent & $7.67(0.72)$ & $6.12(0.72)$ & $4.13(1.14$ & 5.58 \\
No Perfume & $6.72(1.07)$ & $5.90(0.83)$ & $4.30(1.29)$ \\
Total & $7.49(1.21)$ & $6.29(1.13)$ & \\
\hline
\end{tabular}


between self-rated attractiveness and provided ratings of attractiveness for the facial stimuli. A MANOVA indicated no significant effect of week of cycle on ratings of attraction. A second MANOVA concerned with the influence of relationship status on ratings revealed no significant effect of this variable.

\section{Discussion}

The analysis of the data revealed some interesting findings. Firstly, the pilot study identification of facial stimuli rated as low, medium and high in attractiveness was clearly successful as the main effect for the ratings of attractiveness given here indicates a clear difference between the three categories. This provides validation that any subsequent effects of category of stimulus can be attributed to differences in attractiveness. The lack of a significant effect of perfume type on ratings of attractiveness was not predicted and suggests that any impact of perfume type on any of the halo characteristics may not be mediated solely by an increase in perceived attractiveness of the stimuli. The significant interaction between stimulus category and perfume condition might suggest that the impact of perfume on ratings of attraction for the differing categories might be more complex than first envisaged. However, the analysis of simple effects (as presented in Figure 1) identified that the only significant increase in attractiveness as a consequence of perfume type is when comparing the congruent and incongruent perfumes for the medium attractive stimuli. The comparison between the congruent and no perfume conditions in this category approached but did not reach statistical significance. It might be expected that the medium category would be most available for change of ratings, sitting as it does in the middle of the scale and therefore with headroom for modulation in both directions. However, given that the use of perfumes is based upon increasing attractiveness, it could be argued that the low attractiveness category could be the one where most effect might be noticed given the initial low baseline. The lack of any such effect perhaps indicating that the strength of the visual stimuli overwhelmed any possible impact of perfume in terms of ratings of attractiveness.

As might be predicted strong significant effects of attractiveness category were found for each halo effect variable with the more attractive stimuli gaining the most positive ratings. Existing literature has identified an attractiveness-induced halo effect concerning intelligence [6] and trustworthiness (which can be seen as a proxy for reliable; [8]). Strong links with perceived outgoingness, sincerity, extraversion and friendliness are also commonly reported [7]. Whilst social competence has not been examined as such, the latter two adjectives can be seen as proxies of the trait. Thus the present findings are consistent with previous reports, endorsing attractiveness as the key predictor of personal evaluations. Given the somewhat disappointing impact of perfume on the measures of attractiveness however, the findings for the effect perfume condition on the halo effect variables are even more striking. With the exception of the "Reliable" variable, significant main effects of perfume were found for each of these variables with the congruent male perfume producing the highest ratings, and the incongruent female perfume often producing ratings that did not differ significantly to the no perfume condition. The clear conclusion that might be drawn from this is that congruency is important when selecting perfume if it is to be employed to facilitate favourable first impressions, even if these might not extend to how attractive one is perceived.

So, if the impact of perfumes is not directly on attractiveness and the effects of perfume and attractiveness are somewhat independent when it comes to induced halo effects, how might we explain the congruency effect? Consideration of the hedonic valence hypothesis leads to its early rejection here. The rate at which an individual may become aware of a smell depends on its hedonic quality, such that foul smells are detected notably faster, produce more rapid behavioural responses [28] [29], and less than favourable evaluations of face stimuli [30]. Pleasant smells do not evoke this rapid response and prolonged exposure is required for an overt effect to be observed. As Kirk-Smith and Booth established, such scents have an indirect impact on participants' mood, so favourable judgements emerge as a by-product [24] [26]. Although mood was not measured in the current study, the notion that the incongruent (female) perfume was considered unpleasant seems implausible given the successful commercial nature of the product. As such the difference between the congruent and incongruent perfume conditions is unlikely to be a consequence of mood changes based on the pleasantness of the perfume mediating the effects observed for ratings of the halo variables. To provide further evidence regarding this, future research should administer a mood rating scales before and after completion of the study,and include a measure of odour pleasantness. Interestingly, Capparuccini and colleagues investigated the impact of hedonic valence and failed to observe a halo effect for sexually-neutral characteristics of the kind employed here [27]. A plausible explanation for this discrepancy might be found in the congruence of the perfumes they employed, especially 
as they were applied to stimuli of a range of ages and for both genders in a repeated measures design. The current study clearly indicates that a gender-congruent perfume can produce a halo effect for a range of sexually neutral characteristics, whereas an incongruent one does not. The question remains as to why this effect has been observed?

Findings from research in the arena of food and product preference might provide some suggestions. We generally see products and foods before smelling and/or eating them. Early visual cues convey sensory impressions and may generate strong expectations, especially for olfactory perception, [31]-[33]. According to the assimilation-contrast theory [34], visual information is subsequently confirmed or disconfirmed through the smelling or eating experience of the product itself [35]. Expected and actual experiences are therefore combined to produce the final evaluation of the stimulus. Zellner's group showed that congruent visual-odourdrink combinations are rated more pleasant, easier to identify, and consequently increasing the positive hedonic value [36]. Zellner and Durlach studied the influence of colour-flavour congruence on the expected and experienced refreshment, intensity, and liking of beverages. Lemon and mint solutions coloured brown were rated as less refreshing and less liked, than when paired with congruent colours [37]. In the same vein, it has beenreported that congruence between colour and odour was important for determining the freshness of soft drinks, dishwashing liquids and scented candles [38]. Applying a reverse version of the assimilation-contrast theory to the findings of the current study might suggest that the ambient male oriented perfume produced an expectation of male visual stimuli and that this pairing led to enhanced evaluations once the stimuli presentation took place. However, although pleasant in itself, the incongruent female perfume might produce the expectation of female visual stimuli and the incongruence of this expectation when paired with male stimuli might, as observed, reduce ratings to a level equal to that when no perfume is presented.

The slightly uneven distribution of the halo effect is not well explained through this reasoning, but consideration of other factors can help. The non-significant effect for the "Reliable" variable might indeed be a consequence of sample size, with the effect size here being larger than those often found for the impact of aromas on other aspects of cognition and mood e.g. [39]-[41]. Certainly, consideration of the mean values indicates differences that in terms of direction are in line with the significant effects found for the other halo effect variables, but with a smaller effect size present. Perhaps it might be a consequence of this variable always being presented first in the list that produced the smaller effect. Future research should consider balancing the presentation of the dependent variables in order to prevent any such possibility. When considered in these terms the failure of the congruent perfume to impact significantly on ratings of attractiveness might also be explained as this measure was taken for each stimulus prior to completion of the halo variables scales. These possibilities are worthy of further investigation. The relatively small effect for the "Wealth" variable which appeared later in the series of variables to rate might be attributed to the fact that it is not a personality attribute per se; instead it is a materialistic concept that might be a proxy for life success. The abundance of research support for beauty-generated halo effects has covered intrinsic factors directly related to the persona. And although literature has suggested personality can be derived from the face [42], the extrinsic nature of the "Wealth" variable might make this less available for inflation. However, data previously reported have demonstrated that attractiveness is directly proportional to perceived Socio Economic Status-a concept closely related to wealth [43].

Consistent with Berliner's results indicating a pheromone-generated halo effect in a female sample [19], the current study has demonstrated that commercially available sex-congruent perfumes can produce a similar effect. This emphasizes the role of olfactory cues-including those with no biological significance-amongst women. Males have been shown to be equally affected by pheromones, but their response to synthetic smells must be tested comprehensively too, to see if similar effects to those reported here will be found, despite males naturally lower threshold for olfactory cues [44]. Additional sex differences that go beyond female superiority in smell perception also present opportunities for further investigation. Firstly, women assign more importance to smell in mate selection; conversely, men base such decisions primarily on visual cues [45]. This suggests that olfactory sensory information may go largely unnoticed by male participants, implying that responses to smell by men and women may be of different nature and magnitude [46]. Secondly, a phenomenon known as "female positivity" referring to womens' tendency to assign positive traits to others, due to their nurturing and socially sensitive nature may be evident in the current data [47]. Whether this phenomenon is independent of olfactory modulation would make an interesting investigation.

A major procedural issue concerning much olfactory-related research is the lack of alternation between scents. Due to the nature of the design of the present study, this limitation could not have been addressed, and an effect 
known as "olfactory fatigue" may have resulted from it. Olfactory fatigue is the sensory habituation to olfactory stimuli following stimulation presented at a constant level for as little as a few seconds, resulting in blunting of the sense [48]. This proposal has been supported by the work of Ekman and colleagues, who reported that the intensity of odour perception decreased exponentially as duration of odour presentation increased [49]. Interestingly, when presented to a supposedly neutral/healthy scent, participants showed rapid adaptation, but became more sensitized when they were informed they were in the presence of a hazardous odour [50], suggesting a differential adaptation interval as a result of perceived odour "quality". Future research would benefit from performing pilot studies whereby the length of time taken to habituate to a smell is investigated prior to the experimental phase. Subsequently, the duration of the study should be accommodated to this time frame so as to ensure that perception remains above the threshold throughout the study.

To conclude, the findings reported here indicate that our views and opinions of others can be influenced by facial attractiveness, and furthermore that this influence can be further affected by gender congruent perfume. The finding that the effect of perfume on ratings of a range of personal attributes might be found even if attractiveness is not significantly increased provides a novel outcome to be considered in future research. Overall, results here and elsewhere have potential implications in a wide range of contexts. The correct perfume selection might lead individuals to be rated more positively on a range of characteristics that could increase success in a romantic encounter. Moreover, and potentially more important are the possible effects on events and activities related to life success. For example, perfume generated halo effects could improve the ratings of students in the classroom; could lead candidates to fare better in a job interview, or even the workplace and associated reviews and appraisals. The possible existence of such effects in applied contexts warrants the development of an applied field of research in this area.

\section{References}

[1] VandenBos, G. (2007) APA Dictionary of Psychology. American Psychological Association, Washington.

[2] Bull, R. and Rumsey, N. (1988) The Social Psychology of Facial Appearance. Springer-Verlag, London. http://dx.doi.org/10.1007/978-1-4612-3782-2

[3] Langlois, J., Kalakanis, L., Rubenstein, A., Larson, A., Hallam, M. and Smoot, M. (2000) Maxims or Myths of Beauty? A Meta-Analytic and Theoretical Review. Psychological Bulletin, 126, 390-423. http://dx.doi.org/10.1037/0033-2909.126.3.390

[4] Hamermesh, D.S. (2011) Beauty Pays: Why Attractive People Are More Successful. Princeton University Press, New Jersey.

[5] Thorndike, E. (1920) A Constant Error in Psychological Ratings. Journal of Applied Psychology, 4, 25-29. http://dx.doi.org/10.1037/h0071663

[6] Zebrowitz, L., Hall, J., Murphy, N. and Rhodes, G. (2002) Looking Smart and Looking Good: Facial Cues to Intelligence and Their Origins. Personality and Social Psychology Bulletin, 28, 238-249. http://dx.doi.org/10.1177/0146167202282009

[7] Dion, K., Berscheid, E. and Walster, E. (1972) What Is Beautiful Is Good. Journal of Personality and Social Psychology, 24, 285-290. http://dx.doi.org/10.1037/h0033731

[8] Wilson, R.K. and Eckel, C C. (2006) Judging a Book by Its Cover: Beauty and Expectations in the Trust Game. Political Research Quarterly, 59, 189-202. http://dx.doi.org/10.1177/106591290605900202

[9] Feingold, A. (1992) Good-Looking People Are Not What We Think. Psychological Bulletin, 111, 304-341. http://dx.doi.org/10.1037/0033-2909.111.2.304

[10] Ritts, V., Patterson, M.L. and Tubbs, M.E. (1992) Expectations, Impressions, and Judgments of Physically Attractive Students: A Review. Review of Educational Research, 62, 413-426. http://dx.doi.org/10.3102/00346543062004413

[11] Jackson, L.A., Hunter, J.E. and Hodge, C.N. (1995) Physical Attractiveness and Intellectual Competence: A MetaAnalytic Review. Social Psychology Quarterly, 58, 108-122. http://dx.doi.org/10.2307/2787149

[12] Mulford, M., Orbell, J., Shatto, C. and Stockard, J. (1998) Physical Attractiveness, Opportunity, and Success in Everyday Exchange. The American Journal of Sociology, 103, 1565-1592. http://dx.doi.org/10.1086/231401

[13] Watkins, L.M. and Johnston, L. (2002) Screening Job Applicants: The Impact of Physical Attractiveness and Application Quality. International Journal of Selection and Assessment, 8, 76-84. http://dx.doi.org/10.1111/1468-2389.00135

[14] Hosoda, M., Stone-Romero, E. and Coats, G. (2003) The Effects of Physical Attractiveness on Job-Related Outcomes: A Meta-Analysis of Experimental Studies. Personnel Psychology, 56, 431-462. 
http://dx.doi.org/10.1111/j.1744-6570.2003.tb00157.x

[15] Fletcher, J. (2009) Beauty vs Brains: Early Labor Market Outcomes of High School Graduates. Economics Letters, 105, 321-325. http://dx.doi.org/10.1016/j.econlet.2009.09.006

[16] Grosser, B.I., Monti-Bloch, L., Jennings-White, C. and Berliner, D.L. (2000) Behavioral and Electrophysiological Effects of Androstadienone, a Human Pheromone. Psychoneuroendocrinology, 25, 289-299. http://dx.doi.org/10.1016/S0306-4530(99)00056-6

[17] Kirk-Smith, M. and Booth, D. (1980) Effects of Androstenone on Choice of Location in Other's Presence. In: Van der Starre, H., Ed., Olfaction and Taste VII, IRL Press, London, 397-400.

[18] Gustavson, A.R., Dawson, M.E. and Bonett, D.G. (1987) Androstenol, a Putative Human Pheromone, Affects Human (Homo sapiens) Male Choice Performance. Journal of Comparative Psychology, 101, 210-212. http://dx.doi.org/10.1037/0735-7036.101.2.210

[19] Berliner, D.L. (1994) Fragrance Compositions Containing Human Pheromones. United States Patent Office No. 5278141.

[20] Soars, B. (2009) Driving Sales through Shoppers' Sense of Sound, Sight, Smell and Touch. International Journal of Retail \& Distribution Management, 37, 286-298. http://dx.doi.org/10.1108/09590550910941535

[21] Spangenberg, E.R., Sprott, D.E., Grohmann, B. and Tracy, D.L. (2006) Gender-Congruent Ambient Scent Influences on Approach and Avoidance Behaviors in a Retail Store. Journal of Business Research, 59, 1281-1287. http://dx.doi.org/10.1016/j.jbusres.2006.08.006

[22] Waskul, D.D. and Vannini, P. (2008) Smell, Odor, and Somatic Work: Sense-Making and Sensory Management. Social Psychology Quarterly, 71, 53-71. http://dx.doi.org/10.1177/019027250807100107

[23] Hyde, J. and DeLamater, J.D. (2011) Understanding Human Sexuality. 11th Edition, McGraw-Hill, New York.

[24] Berliner, D., Jennings-White, C. and Lavker, R. (1991) The Human Skin: Fragrances and Pheromones. The Journal of Steroid Biochemistry and Molecular Biology, 39, 671-679. http://dx.doi.org/10.1016/0960-0760(91)90266-8

[25] Baron, R. (1981) Olfaction and Human Social Behaviour: Effects of a Pleasant Scent on Attraction and Social Perception. Personality and Social Psychology Bulletin, 7, 611-616. http://dx.doi.org/10.1177/014616728174016

[26] Kirk-Smith, M.D. and Booth, D.A. (1990) The Effect of Five Odorants on Mood and the Judgments of Others. Chemical Signals in Vertebrates, 5, 48-54.

[27] Capparuccini, O., Berrie, C. and Mazzatenta, A. (2010) The Potential Hedonic Role of Olfaction in Sexual Selection and Its Dominance in Visual Cross-Modal Interactions. Perception, 39, 1322-1329. http://dx.doi.org/10.1068/p6736

[28] Jacob, T.J., Fraser, C., Wang, L., Walker, V. and O’Connor, S. (2003) Psychophysical Evaluation of Responses to Pleasant and Mal-Odour Stimulation in Human Subjects; Adaptation, Dose Response and Gender Differences. International Journal of Psychophysiology, 48, 67-80. http://dx.doi.org/10.1016/S0167-8760(03)00020-5

[29] Gostelow, P., Parsons, S.A. and Stuetz, R.M. (2001) Odour Measurements for Sewage Treatment Works. Water Research, 35, 579-597. http://dx.doi.org/10.1016/S0043-1354(00)00313-4

[30] Dematte, M., Osterbauer, R. and Spence, C. (2007) Olfactory Cues Modulate Facial Attractiveness. Chemical Senses, 32, 603-610. http://dx.doi.org/10.1093/chemse/bjm030

[31] Cardello, A. (1994) Consumer Expectations and Their Role in Food Acceptance. In: MacFie, H. and Thomson, D., Eds., Measurement of Food Preference, Elsevier, London, 253-297. http://dx.doi.org/10.1007/978-1-4615-2171-6_10

[32] Dalton, P. (2002) Olfaction. In: Yantis, S. and Pashler, H., Eds., Steven's Handbook of Experimental Psychology: Vol. 1. Sensation and Perception, 3rd Edition, John Wiley, New York, 691-746.

[33] Demattè, M., Sanabria, D. and Spence, C. (2006) Cross-Modal Associations between Odors and Colors. Chemical Senses, 31, 531-538. http://dx.doi.org/10.1093/chemse/bjj057

[34] Anderson, R. (1973) Consumer Dissatisfaction: The Effect of Disconfirmed Expectancy on Perceived Product Performance. Journal of Marketing Research, 10, 38-44. http://dx.doi.org/10.2307/3149407

[35] Deliza, R. and MacFie, H. (1996) The Generation of Sensory Expectation by External Cues and Its Effect on Sensory Perception and Hedonic Ratings: A Review. Journal of Sensory Studies, 11, 103-128. http://dx.doi.org/10.1111/j.1745-459X.1996.tb00036.x

[36] Zellner, D., Bartoli, A. and Eckard, R. (1991) Influence of Color on Odor Identification and Liking Ratings. American Journal of Psychology, 104, 547-561. http://dx.doi.org/10.2307/1422940

[37] Zellner, D. and Durlach, P. (2003) Effect of Color on Expected and Experienced Refreshment, Intensity, and Liking of Beverages. American Journal of Psychology, 116, 633-647. http://dx.doi.org/10.2307/1423663

[38] Fenko, A., Schifferstein, H., Huang, T. and Hekkert, P. (2009) What Makes Products Fresh: The Smell or the Colour? 
Food Quality and Preference, 20, 372-379. http://dx.doi.org/10.1016/j.foodqual.2009.02.007

[39] Moss, M.C., Cooke, J., Wesnes, K. and Duckett, P. (2003) Aromas of Rosemary and Lavender Essential Oils Differentially Affect Cognition and Mood in Healthy Adults. International Journal of Neuroscience, 113, 15-38. http://dx.doi.org/10.1080/00207450390161903

[40] Moss, M., Hewitt, S., Moss, L. and Wesnes, K. (2008) Modulation of Cognitive Performance and Mood by Aromas of Peppermint and Ylang-Ylang. International Journal of Neuroscience, 118, 59-77. http://dx.doi.org/10.1080/00207450601042094

[41] Moss, L., Rouse, M., Wesnes, K.A. and Moss, M. (2010) Differential Effects of the Aromas of Salvia Species on Memory and Mood. Human Psychopharmacology: Clinical and Experimental, 25, 388-396. http://dx.doi.org/10.1002/hup.1129

[42] Pton-Voak, I.S., Pound, N., Little, A.C. and Perrett, D.I. (2006) Personality Judgments from Natural and Composite Facial Images: More Evidence for a "Kernel of Truth" in Social Perception. Social Cognition, 24, 607-640. http://dx.doi.org/10.1521/soco.2006.24.5.607

[43] Kalick, S.M. (1988) Physical Attractiveness as a Status Cue. Journal of Experimental Social Psychology, 24, $469-489$. http://dx.doi.org/10.1016/0022-1031(88)90047-9

[44] Doty, R.L. and Cameron, E.L. (2009) Sex Differences and Reproductive Hormone Influences on Human Odor Perception. Physiology \& Behavior, 97, 213-228. http://dx.doi.org/10.1016/j.physbeh.2009.02.032

[45] Havlicek, J., Saxton, T.K., Roberts, S.C., Jozifkova, E., Lhota, S., Valentova, J. and Flegr, J. (2008) He Sees, She Smells? Male and Female Reports of Sensory Reliance in Mate Choice and Non-Mate Choice Contexts. Personality and Individual Differences, 45, 565-570. http://dx.doi.org/10.1016/j.paid.2008.06.019

[46] Herz, R.S. and Inzlicht, M. (2002) Sex Differences in Response to Physical and Social Factors Involved in Human Mate Selection: The Importance of Smell for Women. Evolution \& Human Behavior, 23, 359-364. http://dx.doi.org/10.1016/S1090-5138(02)00095-8

[47] Winquist, L.A., Mohr, C.D. and Kenny, D.A. (1998) The Female Positivity Effect in the Perception of Others. Journal of Research in Personality, 32, 370-388. http://dx.doi.org/10.1006/jrpe.1998.2221

[48] Moncrieff, R.W. (1956) Olfactory Adaptation and Odour Likeness. The Journal of Physiology, 133, 301-316.

[49] Ekman, G., Berglund, B., Berglund, U. and Lindvall, T. (2008) Perceived Intensity of Odor as a Function of Time of Adaptation. Scandinavian Journal of Psychology, 8, 177-186. http://dx.doi.org/10.1111/j.1467-9450.1967.tb01392.x

[50] Dalton, P. (1996) Odor Perception and Beliefs about Risk. Chemical Senses, 21, 447-458. http://dx.doi.org/10.1093/chemse/21.4.447 
Scientific Research Publishing (SCIRP) is one of the largest Open Access journal publishers. It is currently publishing more than 200 open access, online, peer-reviewed journals covering a wide range of academic disciplines. SCIRP serves the worldwide academic communities and contributes to the progress and application of science with its publication.

Other selected journals from SCIRP are listed as below. Submit your manuscript to us via either submit@scirp.org or Online Submission Portal.
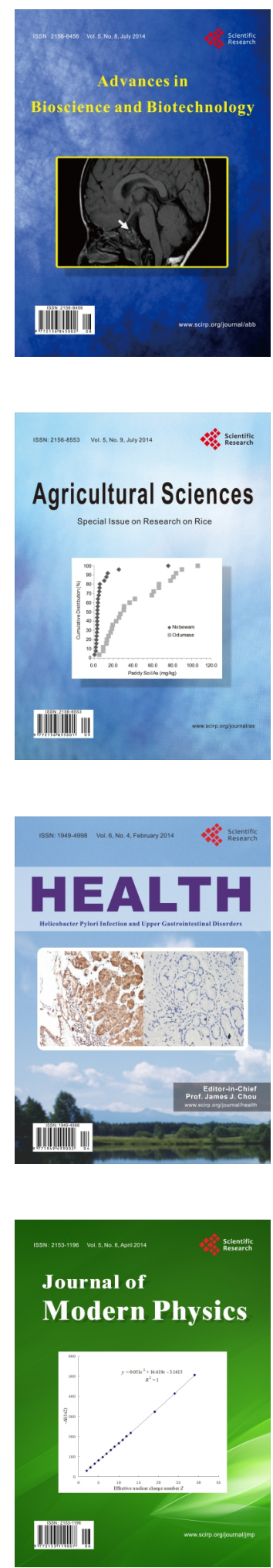
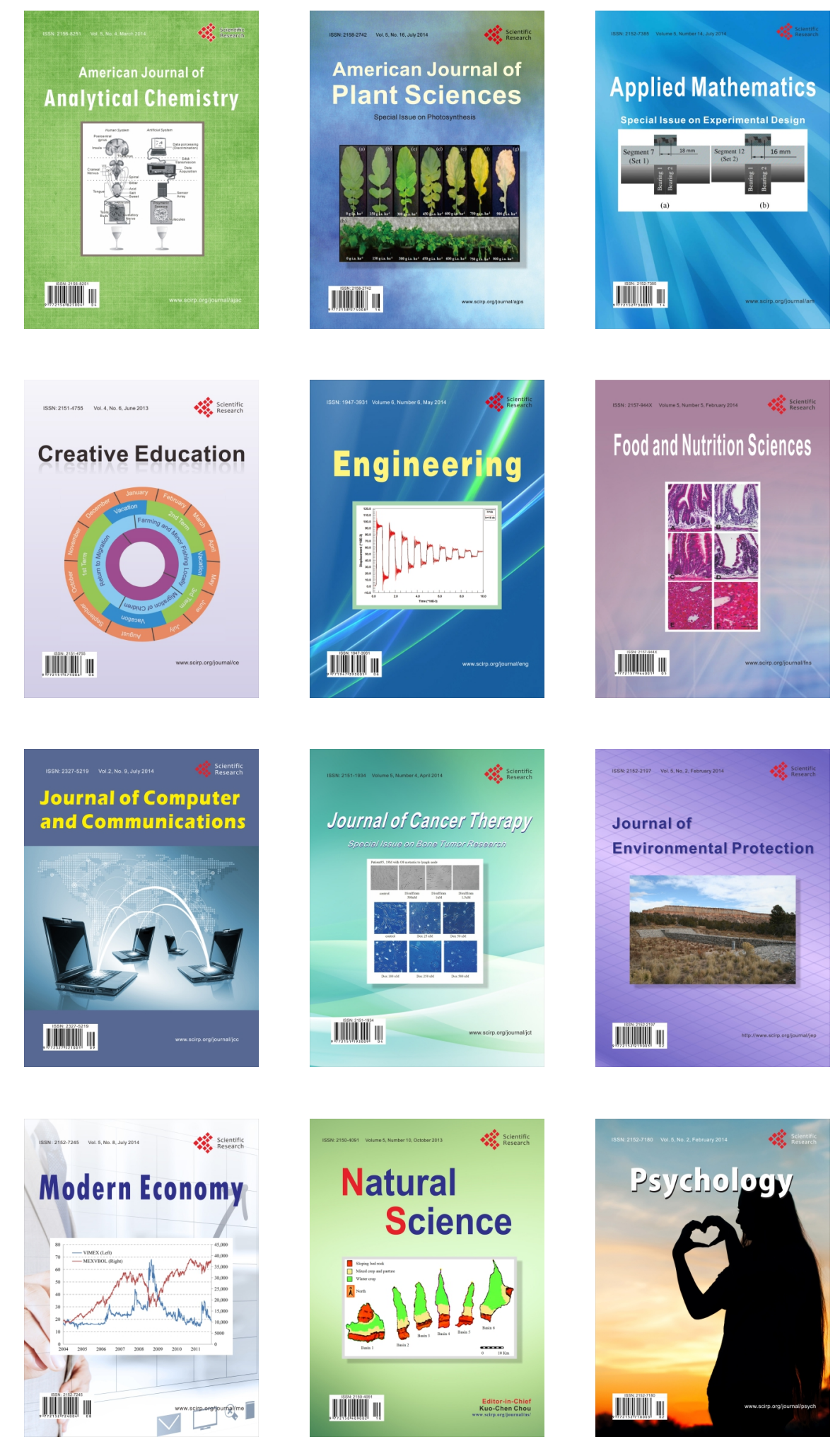